\title{
PROPOSTA DE OTIMIZAÇÃO DO USO DE BROMIDRATO DE FENOTEROL GOTAS PARA PRÁTICA INALATÓRIA
}

\author{
Cristiano Guilherme Alves de OLIVEIRA ${ }^{1}$, Eduardo SHIMODA ${ }^{2 *}$, Marcella Costa RADAEL ${ }^{2}$ \& Aldo \\ SHIMOYA ${ }^{2}$.
}

${ }^{1}$ Universidade Iguaçu - Campus V, Itaperuna, Rio de Janeiro, Brasil.
${ }^{2}$ Universidade Candido Mendes - Campos dos Goytacazes, Rio de Janeiro, Brasil.
*Autor para correspondência: shimoda@ucam-campos.br

http://dx.doi.org/10.18571/acbm.094

\section{RESUMO}

Bromidrato de fenoterol é um fármaco broncodilatador usado no tratamento de doenças pulmonares obstrutivas, sendo amplamente empregado no sistema de saúde pública em inaloterapia de crianças e adultos com pneumopatias. Porém, o peso de uma gota pode variar, resultando na possibilidade de administração inadequada. Portanto, objetivou-se estudar a variabilidade do tamanho das gotas do medicamento, identificando as frequências relativas de subdosagens e sobredosagens. Aferições do peso das gotas foram realizadas com auxílio de balança analítica com precisão de décimos de miligrama $(0,0001 \mathrm{~g})$. O frasco com dispositivo gotejador foi posicionado com inclinação formando um ângulo de $90^{\circ}$ em relação à superfície e o medicamento pesado gota a gota. Tais gotas foram agrupadas de 2 a 2 até 27 a 27, sendo realizada análise do coeficiente de variação dos agrupamentos. Considerando-se a dosagem ideal do medicamento recomendado pela literatura e a margem aceitável de variação $( \pm 10 \%)$, foram determinadas as frequências de subdosagens, dosagens aceitáveis e sobredosagem nos diversos agrupamentos. Resultados demonstram que a variabilidade dos pesos das gotas diminui com o aumento do número de gotas agrupadas. A maior frequência estimada de gotas com peso aceitável ocorreu quando estas foram agrupadas de 20 a 20. As menores frequências estimadas de subdosagem e sobredosagem ocorreram em grupos com 15 e 25 unidades, respectivamente. Conclui-se que, para o processo de otimização, há a necessidade da constituição de uma "solução de trabalho" com valores de 20 gotas ou $1 \mathrm{~mL}$ de fenoterol, em que os efeitos de overdose e subdoses terapêuticas são minimizados.

Palavras chave: Bromidrato de Fenoterol; Conta-gotas; Qualidades Estatísticas.

\begin{abstract}
Fenoterol hydrobromide is a bronchodilator drug used in treatments of obstructive pulmonary diseases, widely applied on the public health system in inhalotherapy for children and adults with pulmonary disease. However, the drop weight may vary, having as a consequence the possibility of improper administration. Therefore, this study aims at verifying the variability of the drop size of the medicine, identifying the relative frequencies of underdose and overdose. Measurements of the weight of the drops were carried out with the aid of an analytical balance with accuracy of tenths of milligram $(0.0001 \mathrm{~g})$. The medicine bottle with dripper device was positioned at an angle of $90^{\circ}$ to the surface and the medicine was weighed drop by drop. These drops were grouped of 2 in 2 up to 27 in 27, performing analysis of the variation coefficient of the groupings. Considering the optimal dosing of the recommended medicinal product by literature and the acceptable margin of variation $( \pm 10 \%)$, the frequencies of underdoses, acceptable doses and overdoses in the various groupings were determined. Results show that the variability of the weights of the drops decreases with increasing number of drops grouped. The greater frequency estimated of drops with acceptable weight occurred when they were grouped
\end{abstract}




\section{Biomedica Brasiliensia}

ISSN: 2236-0867

of 20 in 20. The lower frequencies estimated of underdose and overdose occurred in groups with 15 and 25 units respectively. It is concluded that, for the optimization process, there is the necessity of the constitution of a "work solution" with values of 20 drops or $1 \mathrm{~mL}$ of fenoterol, in which the effects of underdose and overdose therapeutic treatments are minimized.

Keywords: Fenoterol Hydrobromide; Dropper; Statistical Qualities.

\section{Introdução}

Discussões sobre a qualidade dos medicamentos tem se tornado fator preponderante nos órgãos de saúde do Brasil, assim como nos demais países. Desta forma, surge a farmacoeconomia como área de estudo que possui objetivo de dotar a saúde de ferramentas de tomada de decisão que permitam distribuir os recursos de forma igualitária e ética com maior benefício social (VERANO; MASIS, 2006).

Estudos de avaliação farmacoeconômica incluem a área da metrologia, que mede e compara alternativas farmacêuticas em função de seus custos e benefícios, com o intuito de otimizar os custos de tratamentos e diminuir o acometimento de reações adversas a medicamentos com overdoses ou uso de subdoses com tratamentos ineficazes (ARRUDA; OLIVEIRA, 2009).

Segundo o Instituto Nacional de Metrologia (INMETRO), não existe controle quanto ao tamanho e volume dos utensílios domésticos, como colheres, copos, etc. Devido à imprecisão das medidas provenientes destes utensílios domésticos, copos dosadores, seringas e conta-gotas são considerados alternativos às medidas caseiras, sendo amplamente empregados na administração de medicamentos líquidos (CUNHA et al., 2010).

Entretanto, nas diversas formas de doseamento, verifica-se que uma parcela significativa da população não administra os fármacos na concentração adequada, mesmo quando na forma de gotas (SOBHANI et al., 2008) que representam uma forma de fácil diluição e administração das doses principalmente em pediatria, mas necessitam de otimização no processo de fabricação de gotejadores (YIN et al., 2010). Em adição, a gota por si só não representa uma massa ou volume específico, visto que o volume das gotas de diferentes líquidos varia muito entre si (SKLUBALOVA e ZATLOUKAL, 2006).

Grande parte dos trabalhos relatados sobre controle e uso de gotas referem-se à colírios e em tais medicamentos observou-se que o peso das gotas tem influência da marca do medicamento (PRATA; PRATA JÚNIOR, 2004) e do ângulo de gotejamento (ESTACIA; TOGNON, 2008).

Dentre os medicamentos utilizados no Brasil que são ministrados através de conta-gotas pode ser citado o bromidrato de fenoterol. Este fármaco é utilizado no tratamento de doença pulmonar obstrutiva crônica, alergias respiratórias, asma, bronquite, bronqueolite e infecções virais respiratórias, sendo que o uso contínuo previne a recorrência do quadro clínico pulmonar (ZANNONI; PALHARES, 2002).

$\mathrm{O}$ fenoterol é uma mistura de enantiomeros com formula química de $\mathrm{C}_{17} \mathrm{H}_{21} \mathrm{NO}_{4} \mathrm{HBr}$ e peso molecular 384,3, estão descritos na Farmacopéia Britânica como solução oral nas formas de sais de bromidrato comercializados na forma de gotas e xaropes e aerossol dosificador (ZAMUNER; CARRION; MAGALHÃES, 2008).

A posologia do fenoterol consiste, inicialmente, na diluição do medicamento mediante a utilização de conta-gotas, seguida de nebulização. Após sua inalação seu efeito é imediato e pode durar por 4-6 horas. No entanto, sua comercialização está proibida nos Estados Unidos e há relatos de casos de mortes devido ao seu uso na Nova Zelândia (HOFFMAN, 2003). No Brasil, $22 \%$ das crianças com quadro asmático são tratadas com fenoterol e a utilização é crescente nessa faixa etária (SANTOS et al., 2012). 
Considerando-se o risco representado por administração de dosagem inadequada do fármaco e os estudos realizados com outros medicamentos que mostram que o volume das gotas varia muito entre si, o presente trabalho possui como objetivo estudar a variabilidade do tamanho das gotas do bromidrato de fenoterol, identificando as frequências relativas de subdosagens e sobredosagens.

\section{Material e Métodos}

O medicamento bromidrato de fenoterol, em uma das suas formas genéricas, foi adquirido em farmácia comunitária localizada no município de Itaperuna, na forma farmacêutica líquida, comercializado em frasco conta-gota contendo $20 \mathrm{~mL}$, com concentração de $5 \mathrm{mg}$ do medicamento por $\mathrm{mL}(1 \mathrm{~mL}=20$ gotas $)$.

Com o auxílio de uma balança analítica Gehaka ${ }^{\circledR}$ AG 200, com precisão de décimos de miligrama $(0,0001 \mathrm{~g})$, o frasco com dispositivo gotejador foi posicionado com uma inclinação formando um ângulo de $90^{\circ}$ em relação à superfície e o medicamento pesado gota a gota totalizando 730 gotas. Foram realizadas pesagens correspondentes a três frascos do medicamento de mesma marca e mesmo lote. Realizou-se a pesagem das gotas em ambiente controlado com temperatura à $25^{\circ} \mathrm{C}$.

Para análise estatística dos resultados, utilizou-se o aplicativo Sistema para Análises Estatísticas e Genéticas (SAEG, versão 9.1).

Uma vez tabulados os pesos individuais das gotas, foram somados os pesos de gotas em grupos de 2 até 20 gotas. A seguir, foram considerados para análise os pesos agrupados de forma a totalizar 27 observações de cada grupo. Assim, foram obtidos pesos de 27 gotas individuais, 27 dados de gotas agrupadas de 2 em 2, 27 dados de gotas agrupadas de 3 em 3, e assim sucessivamente, até as 27 observações referentes às gotas agrupadas de $20 \mathrm{em} 20$.

A análise estatística consistiu na obtenção de parâmetros estatísticos descritivos (média e coeficiente de variação) em cada grupo. Além disso, foi testada a existência de tendências no coeficiente de variação, conforme aumentava o número de gotas agrupadas. Para tal, foi obtida uma equação de regressão, sendo o modelo estatístico da regressão escolhido, dentre os disponibilizados pelo aplicativo SAEG, mediante análise dos parâmetros da regressão (significância e coeficiente de determinação).

Considerando-se o peso ideal da gota $(0,05 \mathrm{~g})$ e a variação aceitável $( \pm 10 \%$, ou $0,045 \mathrm{~g}$ a $0,055 \mathrm{~g}$ ) foram determinadas as frequências de subdosagem, dosagem aceitável e sobredosagem em cada agrupamento. Foram obtidas, então, equações de regressão que estimam estas frequências em função do número de gotas agrupadas. A partir destas equações, foram determinados os tamanhos dos agrupamentos que minimizaram a frequência de sub e sobredosagem, bem como os que maximizaram a frequência de dosagens aceitáveis.

Para a otimização da utilização do bromidrato de fenoterol foi proposta uma "solução de trabalho" a partir do cálculo da variabilidade média do peso das gotas e a partir dos valores obtidos para as curvas de regressão de gotas aceitáveis, subdosagem e sobredosagem. A solução foi obtida através da diluição da solução de bromidrato fenoterol gotas em solventes como água destilada ou solução fisiológica $0,9 \%$ com a elaboração de uma tabela com a dosagem ideal por faixa de peso do paciente.

\section{Resultados e Discussão}

Os resultados demonstram a distribuição do peso das gotas quando comparadas ao peso médio destas $(0,04916 \pm 0,00068 \mathrm{~g})$. A distribuição das gotas em relação à variação do peso médio gota a gota $0,04994 \mathrm{~g}$ e o limite de $10 \%$ de aceitabilidade reportados por Alcântra et al. (2007) 
produzem valores de $0,044095 \mathrm{~g}$ e $0,053893 \mathrm{~g}$ para gotas aceitáveis, considerando-se subdosagem valores abaixo de $0,044095 \mathrm{~g}$ e sobredosagem valores acima de $0,053893 \mathrm{~g}$.

Diferenças estatísticas significativas foram observadas em testes com gotas de colírios submetidos a ângulos de $45^{\circ}$ e $90^{\circ}$ (ESTACIA e TOGNON, 2008). Diferenças entre peso médio e volumes também foram identificados em colírios de marcas diferentes (PRATA e PRATA JÚNIOR, 2004). No medicamento dimeticona, também já foi verificado variação do peso das gotas em lotes e marcas diferentes (ARRUDA; OLIVEIRA, 2009). Para dipirona líquida, também foi observado estatisticamente que ocorre cerca de $80 \%$ de variabilidade nas gotas com risco de sobredosagem ou subdosagem (GONÇALVES et al., 2009). No estudo descrito, variabilidade das gotas foi verificada no mesmo lote e marca de fenoterol.

As gotas agrupadas em 27 grupos apresentaram um coeficiente de variação representada por uma equação hiperbólica de $\hat{Y}=8,58+20,564$. $X^{-1}$, em que ocorre redução do coeficiente de variabilidade de acordo com o aumento do número de gotas agrupadas, mostrando um ponto de inflexão no limite permitido pela farmacopeia de $10 \%$ no ponto de 15 gotas agrupadas, abaixo de 5 gotas podem apresentar coeficientes acima de $30 \%$ como pode-se observar na Figura 1.

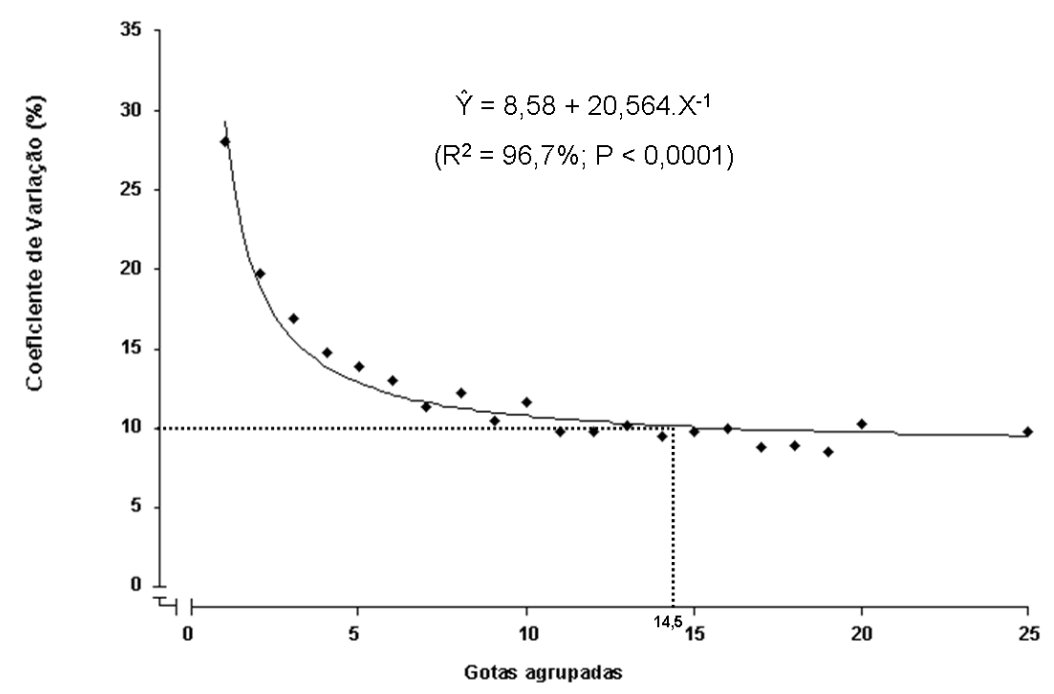

Figura 1: Coeficiente de variação das gotas agrupadas.

Através dos dados da frequência relativa de gotas aceitáveis pela farmacopeia, pode-se obter uma distribuição de gotas aceitáveis e não aceitáveis (subdosagem e sobredosagem), sendo os resultados apresentadas na Figura 2.

As distribuições das frequências relativas das gotas não agrupadas apresentam $57,1 \%$ de aceitabilidade, sendo que os valores de aceitabilidade aumentam com o aumento do número de agrupamentos, verificando-se valores de $81 \%$ de aceitabilidade quando as gotas foram grupos de 19 unidades. As maiores variações do peso médio das gotas do fármaco ocorrem em menores agrupamentos (entre 0 a 9 gotas) com valores que chegam a 19,5\% de subdosagem e 20,6\% de sobredosagem.

Em trabalho com antitérmicos no setor de pediatria do Hospital Santo Antônio de Porto Alegre - RS verificou-se que gotejadores de diversas apresentações disponibilizam volumes/quantidades diversos o que ocasionam erros nas medicações pediátricas disponibilizadas em conta-gotas (SUKIENNIK et al., 2006). Alta variabilidade nas dosagens de medicamentos de uso pediátrico pode implicar em aparecimento de reações adversas, levando a quadros de hospitalizações (SANTOS et al.; 2012). 
Frequência relativa (\%)

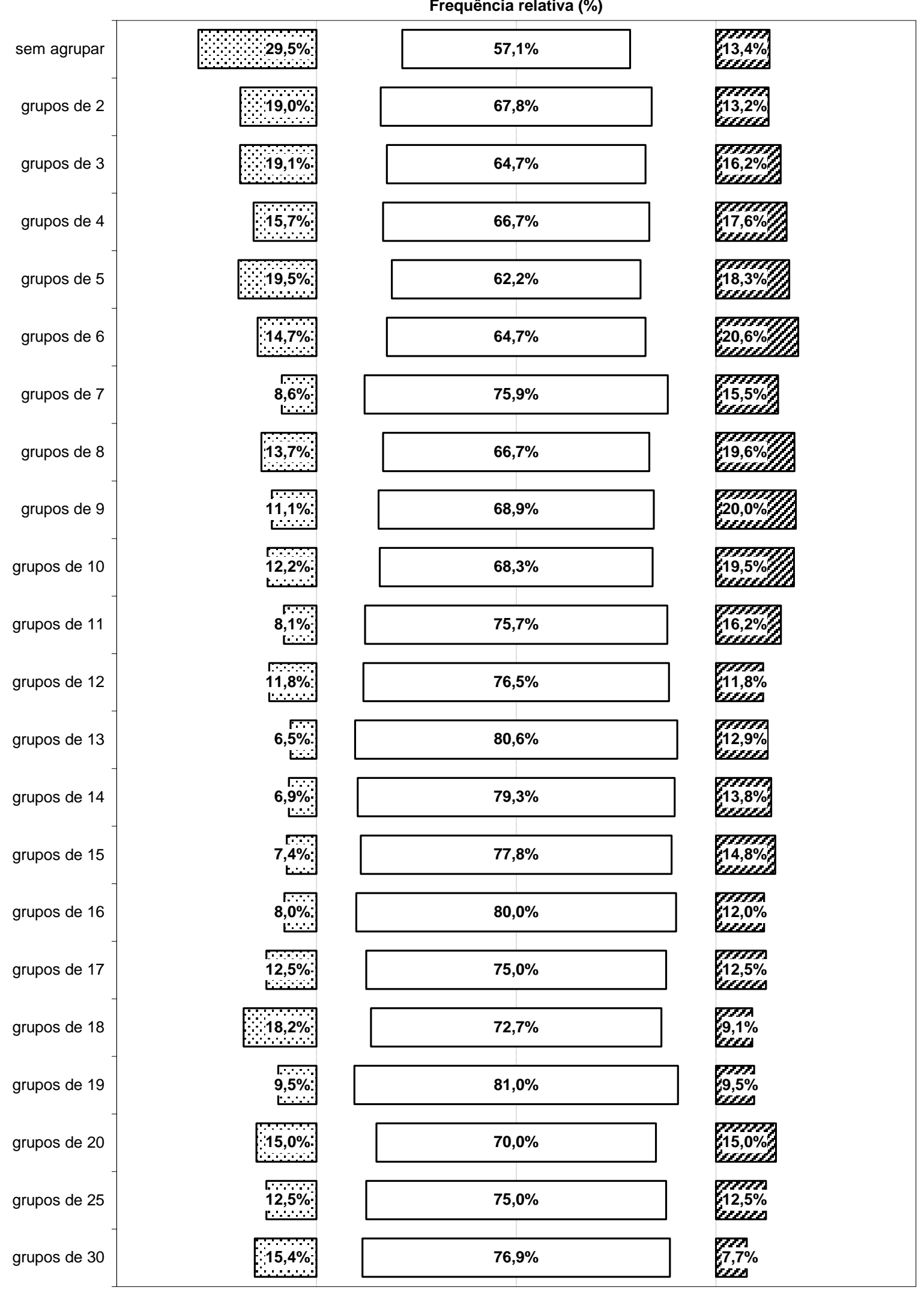

Figura 2: Frequência relativa de gotas agrupadas. 
De acordo com ANVISA e INMETRO, não existem normas técnicas ou regulamentação sobre a utilização de conta-gotas. Em complemento, a ANVISA afirma que "a dosagem oral via conta-gotas segue as informações definidas nos compêndios farmacopeicos como a Farmacopeia Brasileira, que preconiza que as gotas devem ser contadas em conta-gotas normal, escorrendo o líquido livremente". O corpo editorial do Jornal de Pediatria de São Paulo dedica nota aos pediatras nas administrações líquidas e a possível variabilidade nos produtos comercializados, relatando a necessidade de uma ação mais rotineira e de medidas de controle mais eficazes (EJZENBERG, 2006).

Para a análise das gotas agrupadas em relação ao coeficiente de variação e a frequência de subdosagem, foi obtida uma equação de regressão quadrática $\hat{Y}=24,1521-1,76833$. X + $0,0528305 . \mathrm{X}^{2}\left(\mathrm{R}^{2}=58,2 \% ; \mathrm{P}=0,0003\right)$ com ponto de mínima de 15 gotas para os $10 \%$ aceitáveis para a subdosagem.

Os resultados demonstram que em agrupamentos de 2 a 10 gotas, o risco de subdosagem é alto. A bula do medicamento testado indica que, na faixa etária entre 0 a 5 anos, é recomendável a utilização de 2 a 5 gotas, cuja frequência de subdosagem chega a quase $20 \%$.

Segundo Santos et al. (2012) a terapêutica a base de $\beta-2$ agonistas pode resultar num controle inadequado da asma levando ao aumento dos casos de mortes e internações. A utilização de subdoses pode provocar a não redução do quadro de broncoespasmos e o aumento das comorbidades. A idade mais crítica, em que ocorrem maiores incidências de internações por dosagens inadequadas é até os 5 anos de idade.

Para o número de grupamentos aceitáveis, pode-se obter uma equação de regressão quadrática: $\hat{\mathrm{Y}}=59,0540+1,76964 . \mathrm{X}-0,0420472 \cdot \mathrm{x}^{2}\left(\mathrm{R}^{2}=63,6 \% ; \mathrm{P}=0,0001\right)$, como o ponto de máxima em agrupamentos de 20 gotas ou $1 \mathrm{~mL}$. Assim, recomendações de dosagens de 20 gotas seriam aquelas em que haveria maior frequência de administração correta da quantidade indicada.

Para a análise de sobredosagem, foi obtida uma equação de regressão cúbica: $\hat{Y}=12,9869$ $+1,43306 \cdot X-0,129659 \cdot X^{2}+0,00260712 \cdot X^{3}\left(R^{2}=57,5 \% ; P=0,0012\right)$, em que o ponto mínimo foi de aproximadamente 25 gotas. Isto significa que, em casos de recomendação de administração de 25 gotas ocorreria menor frequência de sobredosagens.

Os efeitos tóxicos produzidos pela sobredosagem por $\beta-2$ agonistas inalatórios podem produzir: tremor, taquicardia sinusal, agitação, convulsões, arritmias supraventricular e ventricular, hipocalemia, hiperglicemia e cetoacidose (VALE, 2011). Em condições extremas de envenenamento podem ocorrer alucinações e psicoses.

Zanoni e Palhares (2002), relatam o aparecimento de sonolência, agitação psicomotora, náusea, vomito, taquicardia em pacientes pediátricos sobre a utilização de fenoterol $0,5 \mathrm{mg} / \mathrm{kg}$ gotas. Afirmam ainda que o procedimento inalatório do fármaco deveria ser controlado em ambiente hospitalar com monitorização clínica. Para a utilização em casa recomendam-se outras formas farmacêuticas como aerossóis (MELANI et al.; 2011).

Belela, Pedreira e Peterlini (2011) remetem que cerca de $8 \%$ de erros de medicamentos ligados à pediatria são registrados em bases nacionais e internacionais. Os autores destacam que as crianças são mais suscetíveis e com maior vulnerabilidade devido à ocorrência de fatores intrínsecos, destacando-se características anatômicas e fisiológicas.

Porém, para os mesmos autores, os fatores extrínsecos, como a falta de políticas públicas e a falta de comprometimento da indústria farmacêutica podem levar a uma série de problemas, sendo que há necessidade de implementar estratégias para a diminuição de erros de tratamento na pediatria com eficácia.

A probabilidade de erros em medicações em crianças são cerca de 3 vezes maiores que em adultos. A necessidade do cálculo individualizado da dose, baseada na idade, peso e superfície corpórea da criança, envolvendo múltiplas operações matemáticas em várias fases do processo de medicação (prescrição, dispensação, preparo, administração e monitorização) 
favorece a ocorrência do erro de medicação em crianças (BELELA, PEDREIRA E PETERLINI, 2011).

Estudos concernentes à padronização de doses podem levar a diminuição de quadros de erros de medicação melhorando a eficácia, evitando o agravo de comorbidades e o aumento da segurança com atenuação dos efeitos adversos de sobredosagens (YIN et al., 2010).

Desta forma, estabelece-se a constituição de uma "solução de trabalho" de forma a padronizar as doses de fenoterol gotas para processo de inaloterapia, como forma de otimização da administração de acordo com dados obtidos neste trabalho para as curvas de regressão de gotas aceitáveis, subdosagem e sobredosagem tendo como padrão os pesos médios das gotas agrupadas.

A ANVISA determina que as 20 gotas agrupadas apresentem cerca de $1 \mathrm{~mL}$ do medicamento na apresentação de forma líquida de $20 \mathrm{~mL}$, em que 20 gotas correspondem a 5,0 $\mathrm{mg}$ de fenoterol, sendo 0,25mg por gota (BRASIL, 2012). De acordo com o manual de inaloterapia do consenso brasileiro de asma e manual do hospital da PUC setor de pneumopediatria do hospital Municipal Mario Gati a dose de 1 gota $/ 3 \mathrm{~kg}$, ou seja, $0,25 \mathrm{mg} / 3 \mathrm{~kg}$ $(0,0834 \mathrm{mg} / \mathrm{kg})$ são necessárias para reverter os quadros de pneumopatias relacionadas às broncoconstrição (PUCC, 2012).

Neste, contexto sugere-se o preparo de uma "solução de trabalho", a partir de uma diluição de 20 gotas ou $1 \mathrm{~mL}$ de fenoterol gotas medidos com seringas de $10 \mathrm{~mL}$ acrescidos de 9 $\mathrm{mL}$ de água destilada ou soro fisiológico. Para a otimização das dosagens utilizou-se o cálculo de doses em função do peso de $0,0834 \mathrm{mg} / \mathrm{kg}$ e $1 \mathrm{gt} / 3 \mathrm{~kg}$ com partida inicial às concentrações e volume iniciais da "solução de trabalho" utilizando cálculo de volume final do diluído a ser utilizado por peso com $\mathrm{C}_{\mathrm{i}} \mathrm{V}_{\mathrm{i}}=\mathrm{C}_{\mathrm{f}} \mathrm{V}_{\mathrm{f}}$, obteve-se a Tabela 1 para as dosagens otimizadas por peso da solução de trabalho.

Tabela 1: Relação da dose em função do peso para a solução trabalho de fenoterol líquido $20 \mathrm{~mL}$.

\begin{tabular}{ccc}
\hline Peso $(\mathbf{k g})$ & Dose $(\mathbf{m g})$ & Solução de trabalho $(\mathbf{m L})$ \\
\hline a 3 & 0,25 & 0,5 \\
a 6 & 0,5 & 1,0 \\
7 a 9 & 0,75 & 1,5 \\
9 a 11 & 1,0 & 2,0 \\
12 a 14 & 1,25 & 2,5 \\
15 a 18 & 1,5 & 3,0 \\
10 a 21 & 1,75 & 3,5 \\
21 a 25 & 2,0 & 4,0 \\
\hline
\end{tabular}

Para Rascati (2010) a avaliação da efetividade de qualquer intervenção de assistência à nova saúde, inclusive a de medicamentos, é fundamental para determinar seu papel na prática clínica. Desta forma, a proposta deste trabalho possui a função de produzir uma forma segura na utilização clinica do fenoterol gotas para os quadros de broncoespasmos, em que os níveis de dosimetria possam garantir os $10 \%$ de variabilidade mínima reportada pela farmacopéia.

Cipolla e Gonda (2011) relatam que vários sistemas de nebulização obtêm vários resultados diferentes com inerentes fatores a controlar. Desta forma, há a necessidade de técnicas e medidas na padronização de drogas inalatórias. A elaboração da "Solução de Trabalho" visa à padronização de doses com margem a evitar os efeitos adversos, principalmente os efeitos adrenérgicos cardíacos provocados pelo excesso de droga circulante. Esses efeitos são descritos de forma evidente por Vale (2011) como arritmias supraventriculares e ventriculares.

A otimização das doses com a solução de trabalho permite garantir que a variabilidade mínima do peso médio das gotas não provoque a redução do efeito terapêutico, consequentemente um efeito subclínico com complicações e agravos dos efeitos bronco constritores com aumento do risco de insucesso terapêutico e agravo do quadro clinico do 
paciente. A falha terapêutica pela não conformidade do fenoterol gotas é descrita pelos autores Santos et al. (2012) em trabalho com crianças asmáticas de Salvador, Ba.

Segundo Rascati (2010), a farmacoeconomia mede o benefício trazido por uma intervenção com o custo adicionado ao processo e como este processo apresenta um custoefetividade que permita a mudança de protocolo e procedimentos. O emprego da "Solução de trabalho" traz o mínimo custo para as instituições de saúde sendo requerido para seu manuseio seringa de $10 \mathrm{~mL}$ e solução salina $0,9 \%$ ambos, disponíveis nos serviços de saúde e na pratica médica ambulatorial dos hospitais, assim como nos postos de saúde, prontos-socorros e presente em todas as farmácias comunitárias.

A administração de fenoterol gotas para os quadros de broncoespasmos é recomendada com a utilização de 3 vezes ao dia em inaloterapia e nebulização, permitindo que a diluição da "solução de trabalho" possa ser reutilizada nas demais sessões sem perda do principio ativo quando em solução (BRASIL, 2012).

Desta forma, a "solução de trabalho", representa a otimização do processo de utilização de fenoterol gotas em broncoespasmos para nebulizadores ou administração oral, com uma proposta na mudança de protocolo na adoção de novas técnicas que assegurem a segurança da utilização de fármacos em faixas etárias como idosos e crianças.

Tais intervenções na utilização de técnicas no tratamento de pacientes pediátricos apontam com os aspectos relacionados pelo FDA no Safety and innovation act que trata da necessidade de uma nova abordagem da prescrição de fármacos em populações e faixas etárias órfãs como os idosos e crianças (EUA, 2014).

A proposta da utilização da "solução de trabalho" tem como objetivo trazer à luz as preocupações do Jornal de Pediatria de São Paulo, segundo Sukiennik (2006) e promover debate sobre a resposta enviada a Revista de Pediatria de São Paulo como reportado por Ejzenberg (2006) pelo INMETRO e ANVISA sobre a utilização de medicamentos conta-gotas em crianças.

\section{Conclusões}

Com base nos resultados obtidos, conclui-se que, de acordo com a variabilidade do peso médio das gotas, existe a necessidade de otimização da utilização de fenoterol gotas, no tocante a uniformidade das doses administradas pelo peso do paciente. A maior variabilidade de gotas está ligada a faixa pediátrica de 0 a 9 gotas, sendo suscetíveis ao acometimento de reações adversas provocadas por sobredosagem ou ao aumento da crise de broncoespasmo por subdosagem com aumento das comorbidades da falta de efeito farmacoterapêutico.

Para a otimização da utilização de fenoterol gotas propõe-se a criação de uma solução trabalho com a dose estipulada pelo peso do paciente com $10 \%$ de variabilidade dos pesos médios das gotas assegurando o efeito terapêutico e evitando as reações adversas pertinentes às sobredosagens.

Propondo-se a utilização de métodos estatísticos na qualidade de produtos farmacêuticos na obtenção de doses otimizadas para administração de fármacos de baixo índice terapêutico em conta-gotas. Este trabalho pretende contribuir para uma nova proposta para a avaliação da eficácia terapêutica em populações órfãs onde a seguridade terapêutica traz evidentes benefícios à saúde pública.

\section{Referências}

ALCANTARA G. K. S, VIRTUOSO S, OLIVEIRA S. M. M. Avaliação dos frascos conta-gotas de diferentes marcas de diclofenaco resinato comercializados no mercado brasileiro. Visão Acadêmica (Curitiba); 8:21-6, 2007. 
ARRUDA M. C., OLIVEIRA T. B. Avaliação farmacoeconômica de diferentes marcas de dimeticona comercializada em Anápolis - GO. Visão Acadêmica (Curitiba). 10:106-16, 2009.

BELElA A. S. C., PEDREIRA M. L. G., PETERLINI M. A. S. Erros na Pediatria. Revista Brasileira de Enfermagem (São Paulo). 3: 563-69, 2011.

BRASIL. Farmacopéia Brasileira. 5. ed. Brasilia: Anvisa, 2010. 546 p.

CIPOLLA D. C., GONDA I. Formulation technology to repurpose drugs for inhalation delivery. Drug Discovery Today: Therapeutic Strategies Editors-in-chief (USA). 2011; 3-4.

CUNHA D. A., SIlVA H. J., PERNAMBUCO L. A., MORAES K. J. R., PRADO I. J., ANDRADE G. M., et al. Efeitos da asma no estado nutricional em crianças: Revisão sistemática. Revista Portuguesa de Pneumologia (Portugal). 16: 617-25, 2010.

EJZENBERG B. Respostas do Inmetro e da Anvisa referentes às características dos conta-gotas utilizados nos produtos farmacêuticos. Pediatria (São Paulo). 28: 209-10, 2006.

ESTACIA P., TOGNON T. Considerações sobre o ângulo de administração de colírios antiglaucomatosos análogos das prostaglandinas. Arquivo Brasileiro de Oftalmologia (São Paulo). 5: 684-88, 2008.

EUA. Food Drugs and Administration [Internet]. Safety and Innovation Act. 2014. [Acesso em: 05 outubro 2014]. Disponível em:<http://www.fda.gov/RegulatoryInformation/Legislation/FederalFoodDrugandCosmeticActF DCAct/SignificantAmendmentstotheFDCAct/FDASIA/ucm20027187.htm>.

GONÇALVES A. S., SHIMODA E., PIETROLUONGO M. Proposta de otimização da dosagem de dipirona sódica administrada por conta-gotas. In: Desafios, tendências e perspectivas na educação de engenharia de produção. SIMPEP (Bauru). 1: 1-10, 2009.

HOFFMAN B. B. Catecolaminas, fármacos simpaticomiméticos e antagonistas. In: Gilman AG. As bases farmacológicas da Terapêutica (Rio de janeiro). 10. ed. Macgrow-hill. p. 163-04, . 2003.

MELANI A. S., CANESSA P., COLORETTI I., DEANGELIS G., DETULLIO R., DEL DONNO M., et al. Inhaler mishandling is very common in patients with chronic airflow obstruction and long-term home nebuliser use. Respiratory Medicine. 5: 668-76, 2012.

PRATA JUNIOR J. A., PRATA J. A. Comparação do volume da gota e custo do tratamento dos genéricos de maleato de timolol 0,5\%. Arquivo Brasileiro de Oftalmologia (São Paulo). 67:419-22, 2004.

PUCC (São Paulo). Consenso Brasileiro de Asma. Protocolo para Inaloterapia. Campinas, 2004. [Acesso em: 11 maio 2012]. Disponível em: <http://pt.scribd.com/doc/32991004/39/Protocolopara-Inaloterapia>.

RASCATI K. L. Introdução à Farmacoeconomia. Porto Alegre: Artmed, 2010. 280 p. 
SANTOS D. B., CRUZ A. A., MAGALHÃES S. S., RODRIGUES L. C., CAMARGOS P. A., COELHO H. L., BARRETO ML. Pattern of asthma medication use among children from a large urban center in Brazil. Eur J Clin Pharmacol (Salvador). 69:73-2, 2012.

SKLUBALOVA Z., ZATLOUKAL Z. Study of Eye Drops Dispensing and Dose Variability by Using Plastic Dropper Tips. Drug Development and Industrial Pharmacy (Czech). 32:197-05, 2006.

SOBHANI P., CHRISTOPHERSON J., AMBROSE P. J., CORELLI R. L. Accuracy of Oral Liquid Measuring Devices: Comparison of Dosing Cup and Oral Dosing Syringe. Medication Reviews (New York). 1:46-2, 2008.

SUKIENNIK R., HALPERN R., MANICA J. L. L., PLENTZ F. D., BERGAMIN G., LOSS L., et al. Antitérmicos na emergência pediátrica: estamos usando a dosagem adequada? Jornal de Pediatria (São Paulo). 28: 175-183, 2006.

VALE A. Beta-2 Agonists. Specific Substances. Birmingham. 2011. 108 p.

VERANO R. D., MASIS D. P. Pharmacoeconomy. Acta Medica Colombiana (Bogotá). 2006; 3: 53-5.

YIN H. S., MENDELSOHN A. L, WOLF M. S., PARKER R. M., FIERMAN A., VAN SCHAICK L., et al. Parent`s Medication Administration Errors. American Medical Association (New York). 164:182-6, 2010.

ZAMUNER M. L. M., CARRION R. C. E., MAGALHÃES J. F. Determinação espectrofotométrica do bromidrato de fenoterol em preparações farmacêuticas. Brazilian Journal of Pharmaceutical Sciences (São Paulo). 645-53, 2008.

ZANNONI L., PALHARES D. B. Inalação contínua com fenoterol na criança com asma aguda grave: efeitos clínicos imediatos. Jornal de Pediatria (Rio de Janeiro). 423-28, 2002. 